\title{
Marginal zone B-cell lymphoma mimicking extramedullary plasmacytoma and the clinical outcome after treatment
}

Man Fai Law ${ }^{1,2}$, Hay Nun Chan ${ }^{1}$, Charlotte Leung ${ }^{3}$. Cheuk Kei Wong ${ }^{4}$, Ho Kei Lai ${ }^{1}$, Chung Yin Ha ${ }^{1}$, Celia $\mathrm{Ng}^{1}$, Yiu Ming Yeung ${ }^{1}$, Sze Fai Yip ${ }^{1}$

\author{
${ }^{1}$ Department of Medicine, Tuen Mun Hospital, Hong Kong \\ ${ }^{2}$ Department of Medicine and Therapeutics, Prince of Wales Hospital, Hong Kong \\ ${ }^{3}$ Departments of Pathology, Tuen Mun Hospital, Hong Kong \\ ${ }^{4}$ Departments of Radiology, Tuen Mun Hospital, Hong Kong
}

Submitted: 13 April 2015

Accepted: 21 June 2015

Arch Med Sci 2017; 13, 3: 698-701

DOI: https://doi.org/10.5114/aoms.2017.67290

Copyright (c) 2017 Termedia \& Banach

Marginal zone B-cell lymphoma is occasionally associated with prominent plasma cell differentiation, suggesting a possible histogenic relationship [1]. Primary lymph node plasmacytoma is a rare disorder [2, 3]. It has been suggested to represent an extreme form of plasmacytic differentiation of nodal marginal zone B-cell lymphoma. We report a case of marginal zone B-cell lymphoma with focal plasmacytic differentiation presenting with lymphadenopathy. The lymph node biopsy was initially interpreted as plasmacytoma, and the patient was treated with chemotherapy for plasma cell neoplasm. He had disease progression and then lymph node biopsy was repeated and finally revealed marginal zone B-cell lymphoma. He was treated with chemoimmunotherapy for lymphoma and had a good clinical response.

A 58-year-old man with an unremarkable medical history, except for appendicitis with appendectomy, presented with an enlarged left supraclavicular lymph node of $4 \mathrm{~cm}$ in diameter. He had no fever, weight loss or bone pain. There was no hepatosplenomegaly on physical examination. Complete blood count, liver and renal function tests were unremarkable. Incisional biopsy of the lymph node showed effacement of nodal architecture by a diffuse infiltrate of plasmacytoid cells and immature cells with prominent nucleoli (Figure 1). On immunohistochemical staining, these cells were positive for plasma cell marker CD138 and negative for CD3, CD5, CD20, and CD79a. Kappa light chain restriction was demonstrated. The biopsy result was interpreted as plasmacytoma.

There was an incidental finding of second degree heart block, and a pacemaker was inserted. Echocardiogram showed a large soft tissue mass in the right atrium attached to the atrial septum. Computed tomography (CT) scan of the thorax revealed a large lobulated soft tissue mass occupying the right and left atria with the largest transaxial dimension measuring $7.6 \mathrm{~cm}$. There were mediastinal and left hilar masses representing lymph nodes deposits. The patient was reluctant to undergo biopsy of the intracardiac mass. Bone marrow examination showed active marrow without plasmacytosis. The patient's immunoglobulin G level was $19.7 \mathrm{~g} / \mathrm{l}$, and there was no immunoparesis. The $\beta_{2}$-microglobulin level was $1.85 \mathrm{ng} / \mathrm{ml}$. Skeletal survey did not show any lytic lesions.

\author{
Corresponding author: \\ Man Fai Law \\ Department of Medicine \\ Tuen Mun Hospital \\ Prince of Wales Hospital \\ 852 Shatin, Hong Kong \\ Phone: 852-97763090 \\ E-mail: mflaw99@yahoo. \\ com.hk
}



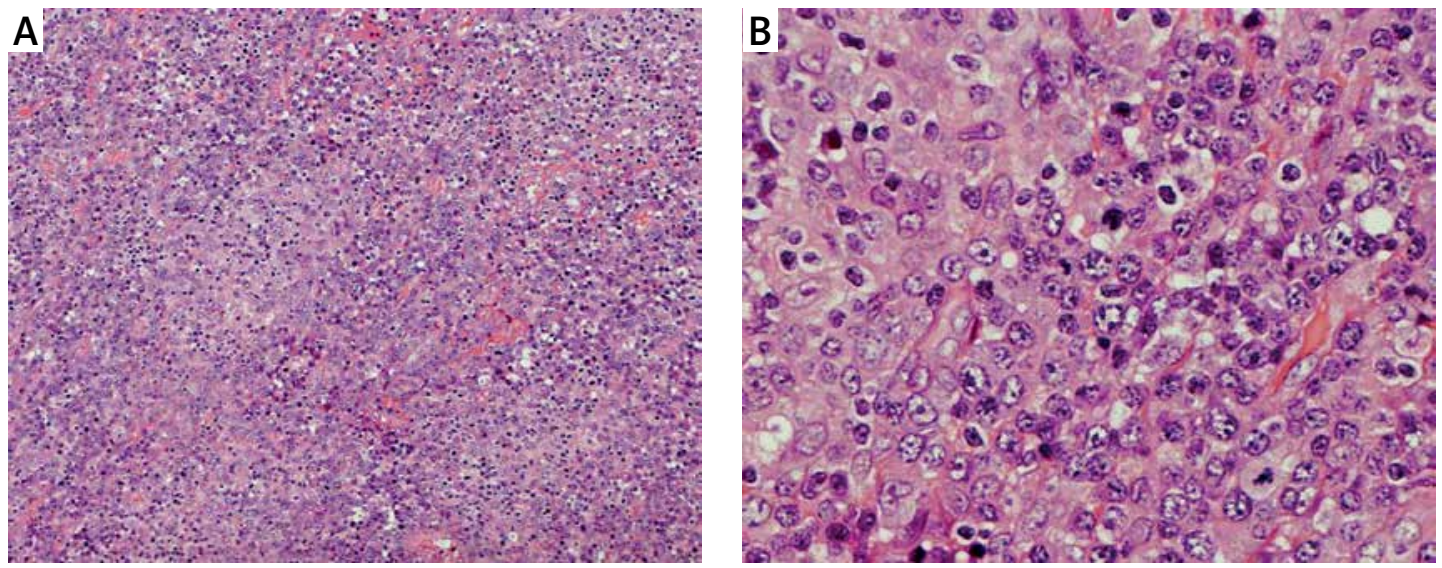

Figure 1. A - Low power field (10x) of the initial lymph node biopsy specimen showing diffuse infiltrates of plasmacytoid cells. B - High power field (40x) of the same specimen showing immature cells with prominent nucleoli

The patient received treatment for extramedullary plasmacytoma. He was given bortezomib (Velcade), thalidomide and dexamethasone (VTD) chemotherapy. The supraclavicular lymph node and intracardiac mass initially decreased in size after two courses of chemotherapy. Further courses of VTD chemotherapy were administered, but this was followed by disease progression, with the left supraclavicular lymph node enlarging to $2 \mathrm{~cm}$ in diameter. Two courses of lenalidomide and dexamethasone were given, but the patient then developed cellulitis at the neck. A course of antibiotics was given, and the cellulitis improved. However, the lymph node rapidly increased in size, causing obstructive symptoms. A CT scan of the neck and thorax showed prominent bilateral cervical lymph nodes and enlarged left supraclavicular lymph nodes, which had increased in size since the previous study.

Supraclavicular lymph node biopsy was repeated, and histological examination showed complete effacement of nodal architecture with diffuse infiltration of abnormal lymphoid cells varying from small to medium-sized lymphoid cells to scattered blast cells. The lymphoid cells had nuclear irregularity and a moderate amount of cytoplasm. Focally, the abnormal lymphoid cells showed plasmacytic differentiation. They also expressed B-cell markers (CD20, CD79a) and were negative for CD5 and CD10. The result was consistent with marginal zone B-cell lymphoma with an increased number of blast cells and focal plasmacytic differentiation.

The first lymph node biopsy was reviewed, and it showed complete effacement of lymph node architecture with extensive infiltration by neoplastic cells which demonstrated plasmacytic differentiation and cytoplasmic $\kappa$ light chain restriction. These plasmacytic cells were CD20 negative, and a small number of $C D 20$ positive B-cells were also found intermixed with the neoplastic plasmacytic cells. It was likely that the previous biopsy had sampled an area of B-cell lymphoma with extensive plasmacytic differentiation and was interpreted as plasmacytoma.

The patient was then given chemoimmunotherapy with rituximab, cyclophosphamide, doxorubicin, vincristine and prednisolone (R-CHOP) in view of the aggressive clinical behavior of the malignancy, and the lymph nodes decreased in size. A total of eight courses of R-CHOP were given, and complete remission was achieved. The patient could tolerate the chemotherapy well, and there were no infective complications. He was still alive four years after therapy.

Lymphoma can resemble plasmacytoma resulting in diagnostic difficulty. Plasmacytic differentiation is not specific to marginal zone B-cell lymphoma. The differential diagnosis may include lymphoplasmacytic lymphoma (LPL) or other B-cell lymphoproliferative disorders with plasmacytic differentiation. Lymphoproliferative disorders (LPDs) associated with plasmacytic differentiation include chronic lymphocytic leukemia (CLL)/small lymphocytic lymphoma (SLL), follicular lymphoma, mantle cell lymphoma (MCL), large cell lymphoma and Burkitt's lymphoma [4]. Integration of the clinical findings with the morphologic, phenotypic, and molecular/cytogenetic findings is important in arriving at the diagnosis.

It is difficult to diagnose nodal marginal zone B-cell lymphoma (NMZL) without the full clinical history, especially when plasmacytic differentiation is present. A differential diagnosis from plasma cell neoplasm can be made by using serum protein electrophoresis to exclude the presence of paraproteinemia [5-7]. Evaluations of bone marrow involvement, anemia, and any lytic bone lesions are performed to exclude other B-cell lymphomas.

Fine cytologic analysis, follicular dendritic cell (FDC) staining by immunohistochemical stains, and knowledge of the existence of phenotypic 
variation (BCL6 expression in large cells, CD5 in some cases) are crucial to recognize NMZL. Flow cytometric analysis and cytogenetic data are also very helpful in some cases. The phenotype is usually CD5-/CD23-/CD10-/BCL6-/cyclin D1- and $\mathrm{BCL} 2+$.

Features that suggest a diagnosis of NMZL are a marginal zone growth pattern, germinal center remnants, follicular colonization, and/or disruption of FDC meshworks in immunohistochemical studies [8]. Ki-67 stains may be used to diagnose transformation to large cell lymphoma, but it is often hampered in NMZL by the presence of numerous germinal center remnants with a high percentage of reactive positive cells admixed with tumor cells colonizing these follicles.

Distinguishing NMZL from LPL is one of the most difficult differential diagnoses in the evaluation of a nodal small B-cell lymphoma with plasmacytic differentiation. The LPL is composed of small lymphocytes, plasmacytoid lymphocytes, and plasma cells, and usually involves the bone marrow and sometimes lymph nodes and spleen. Dutcher bodies, increased mast cells, and hemosiderin are other features. The LPL is often associated with a paraprotein, usually of the IgM type, although the presence of paraprotein is not required for the diagnosis. The lymphoma cells are positive for B-cell antigens by immunohistochemical analysis, and PAX5 coexpression by CD138-positive plasma cells may be another feature [4, 9].

A variety of lymphoproliferative diseases may have plasmacytic differentiation with or without an associated serum paraprotein [10-12]. The $\mathrm{CLL} / \mathrm{SLL}$ is characterized by proliferation of small round lymphocytes interspersed individually with prolymphocytes and paraimmunoblasts together with peripheral blood lymphocytosis. It usually involves nodal or extranodal tissues as diffuse proliferation with pseudofollicular growth centers. Immunoexpression of CD5 and CD23 provide further distinction from MALT lymphoma $[4,8]$.

Follicular lymphoma with plasmacytic differentiation can sometimes be difficult to distinguish from MALT lymphoma. In these cases, plasma cells staining for the same immunoglobulin light chain as the neoplastic follicles are present in the interfollicular areas or within the follicles. Follicular lymphoma is usually positive for CD10 and BCL-6 [13]. The neoplastic follicles are composed exclusively of plasma cells in some rare cases.

The MCL rarely presents with a monotypic plasma cell population; these cases are classified as typical MCL in terms of cytology, histology, and immunohistochemistry [14]. In contrast to MALT lymphoma, MCL cells are typically immunopositive for CD5 and cyclin D1 [4, 15]. Multi-parameter flow cytometry can also be useful in demonstrat- ing clonal plasma cell differentiation in $\mathrm{MCL}$ and distinguishing them from concurrent but unrelated plasma cell neoplasms [16].

Pérez-Galán et al. found that bortezomib resistance in MCL was associated with plasmacytic differentiation, including up-regulation of IRF4 and CD38 and expression of CD138 [17]. In contrast to plasma cells, plasmacytic MCL cells did not increase immunoglobulin secretion. $M C L$ cell lines that were intrinsically bortezomib-resistant expressed plasmacytic features. Plasmacytic differentiation in the absence of an increased secretory load can enable cells to withstand the stress of proteasome inhibition. Expression of CD38 and IRF4 could serve as a marker of bortezomib resistance in MCL [17].

There have been reports of large B-cell lymphoma with plasma cell differentiation $[18,19]$. It is often difficult to differentiate $L P L$ from diffuse large B-cell lymphoma (DLBCL) because some LPLs have large transformed cells, DLBCL can have plasmacytic differentiation, and there are no clear-cut criteria for the distinction unless there is a sheet of transformed cells [4]. However, a report showed that B-cell receptor gene rearrangement studies can be useful in diagnosing diffuse large B-cell lymphoma with plasmacytic differentiation. Immunoglobulin heavy chain and $\kappa$ light chain gene rearrangement studies detected the same clone in the diagnostic and post-therapy lymph node specimens [20].

Burkitt's lymphoma can also have plasmacytoid differentiation, and it is known to be associated with HIV and closely linked to Epstein-Barr virus infection [21]. The tumor cells in Burkitt's lymphoma are usually medium-sized, and the nuclei are round with finely clumped and dispersed chromatin. The cytoplasm is basophilic and usually contains lipid vacuoles.

Our patient was given bortezomib-based chemotherapy for the treatment of the tumor, and there was a partial response initially. It is possible that bortezomib controlled the plasmacytic component of the lymphoma, while the non-plasmacytic component continued to proliferate in our patient. This could explain why there was shrinkage of the supraclavicular lymph node initially. The lymph nodes increased in size again and the patient was given R-CHOP chemoimmunotherapy after the final diagnosis of marginal zone B-cell lymphoma was made. R-CHOP chemoimmunotherapy is one of the recommended treatment options for marginal zone B-cell lymphoma [22]. He had a sustained response and long survival after the treatment.

This case also demonstrated that lymphoid malignancy diagnoses rely on interpretation of good quality material in the context of other relevant 
clinical information and investigations. Incisional and needle core biopsies do not necessarily provide adequate material, and repeating the tissue biopsy to confirm the diagnosis is recommended if there is suspicion about the diagnosis.

In conclusion, marginal zone B-cell lymphoma may mimic extramedullary plasmacytoma. Clinicians should consider the possibility of misdiagnosis when there is an atypical clinical presentation or disease progression during treatment for plasmacytoma.

\section{Conflict of interest}

The authors declare no conflict of interest.

\section{References}

1. Hussong JW, Perkins SL, Schnitzer B, Hargreaves H, Frizzera G. Extramedullary plasmacytoma, a form of marginal zone lymphoma. Am J Clin Pathol 1999; 111: 111-6.

2. Lin BT, Weiss LM. Primary plasmacytoma of lymph nodes. Hum Pathol 1997; 28: 1083-90.

3. Menke DM, Horny HP, Griesser H, et al. Primary lymph node plasmacytomas (plasmacytic lymphomas). Am J Clin Pathol 2001; 115: 119-26.

4. Lin P, Molina TJ, Cook JR, Swerdlow SH. Lymphoplasmacytic lymphoma and other non-marginal zone lymphomas with plasmacytic differentiation. Am J Clin Pathol 2011; 136: 195-210.

5. Bink K, Haralambieva E, Kremer M, et al. Primary extramedullary plasmacytoma: similarities with and differences from multiple myeloma revealed by interphase cytogenetics. Haematologica 2008; 93: 623-6.

6. Kremer M, Ott G, Nathrath M, et al. Primary extramedullary plasmacytomas and multiple myeloma: phenotypic differences revealed by immunohistochemical analysis. J Pathol 2005; 205: 92-101.

7. Lorsbach RB, Hsi ED, Dogan A, Fend F. Plasma cell myeloma and related neoplasms. Am J Clin Pathol 2011; 136: 168-82.

8. Molina TJ, Lin P, Swerdlow SH, Cook JR. Marginal zone lymphomas with plasmacytic differentiation and related disorders. Am J Clin Pathol 2011; 136: 211-25.

9. George TI, Wrede JE, Bangs CD, Cherry AM, Warnke RA, Arber DA. Low-grade B-cell lymphomas with plasmacytic differentiation lack PAX5 gene rearrangements. J Mol Diagn 2005; 7: 346-51.

10. Rymkiewicz G, Gos M, Błachnio K, et al. Mantle cell lymphoma presenting with paraproteinemia. Med Oncol 2005; 22: 319-23.

11. Yin CC, Lin P, Carney DA, et al. Chronic lymphocytic leukemia/small lymphocytic lymphoma associated with IgM paraprotein. Am J Clin Pathol 2005; 123: 594-602.

12. Lin P, Hao S, Handy BC, Bueso-Ramos CE, Medeiros LJ. Lymphoid neoplasms associated with IgM paraprotein: a study of 382 patients. Am J Clin Pathol 2005; 123: 200-5.

13. Gradowski JF, Jaffe ES, Warnke RA, et al. Follicular lymphomas with plasmacytic differentiation include two subtypes. Mod Pathol 2010; 23: 71-9.

14. Visco C, Hoeller S, Malik JT, et al. Molecular characteristics of mantle cell lymphoma presenting with clonal plasma cell component. Am J Surg Pathol 2011; 35: 177-89.
15. Guggisberg K, Jordan RC. Mantle cell lymphoma of the oral cavity: case series and comprehensive review of the literature. Oral Surg Oral Med Oral Pathol Oral Radiol Endod 2010; 109: 98-104.

16. Naushad H, Choi WW, Page CJ, Sanger WG, Weisenburger DD, Aoun P. Mantle cell lymphoma with flow cytometric evidence of clonal plasmacytic differentiation: a case report. Cytometry B Clin Cytom 2009; 76: 218-24.

17. Pérez-Galán P, Mora-Jensen $H$, Weniger MA, et al. Bortezomib resistance in mantle cell lymphoma is associated with plasmacytic differentiation. Blood 2011; 117: 542-52.

18. Montes-Moreno S, Gonzalez-Medina AR, Rodriguez-Pinilla SM, et al. Aggressive large B-cell lymphoma with plasma cell differentiation: immunohistochemical characterization of plasmablastic lymphoma and diffuse large B-cell lymphoma with partial plasmablastic phenotype. Haematologica 2010; 95: 1342-9.

19. Inaba T, Nagata K, Maruyama K. Primary intraocular large B cell lymphoma with plasmacytic differentiation. Int J Hematol 2012; 96: 399-400.

20. Alapat DV, Ramos JM, Anderson J, Post GR. The utility of B-cell receptor gene rearrangement studies in diagnosing diffuse large B-cell lymphoma with plasmacytic differentiation. Ann Clin Lab Sci 2015; 45: 79-82.

21. Carbone A, Cesarman E, Spina M, Gloghini A, Schulz TF. HIV-associated lymphomas and gamma-herpesviruses. Blood 2009; 113: 1213-24.

22. Dreyling M, Thieblemont C, Gallamini A, et al. ESMO Consensus conferences: guidelines on malignant lymphoma. part 2: marginal zone lymphoma, mantle cell lymphoma, peripheral T-cell lymphoma. Ann Oncol 2013; 24: 857-77. 